\title{
Signal Quality Assessment of f-waves in Atrial Fibrillation
}

\author{
Mikael Henriksson ${ }^{1}$, Andrius Petrènas ${ }^{2}$, Vaidotas Marozas ${ }^{2}$, Frida Sandberg ${ }^{1}$, Leif Sörnmo ${ }^{1}$ \\ ${ }^{1}$ Department of Biomedical Engineering and the Center for Integrative Electrocardiology (CIEL), \\ Lund University, Lund, Sweden \\ ${ }^{2}$ Biomedical Engineering Institute, Kaunas University of Technology, Kaunas, Lithuania
}

\begin{abstract}
Ambulatory ECG recordings are frequently corrupted by artifacts caused by, e.g., muscle activity or moving electrodes, which complicates the analysis of $f$-waves and motivates signal quality assessment to improve the reliability of $f$-wave analysis. Although many methods have been developed for assessing the quality of ECG signals in general, no method deals specifically with $f$-waves. This study proposes a novel signal quality index (SQI), using a modelbased approach for assessment of $f$-wave signal quality. To evaluate the performance of the SQI, 189 5-s recordings of $f$-waves from $A F$ patients are studied, as is the same number of recordings with motion artifacts and electrode movements taken from the MIT-BIH Noise Stress Test Database. The signal quality index is capable of discriminating between $f$-waves and noisy recordings with an accuracy of $98 \%$. The results suggest that the proposed signal quality index correctly identifies noisy recordings, and can be used to improve the reliability of $f$-wave analysis.
\end{abstract}

\section{Introduction}

When analyzing ECG signals, it is essential to use a signal quality index (SQI) indicating to what degree the recorded information can be relied on; otherwise, noisy measurements will disrupt the subsequent analysis. This is of particular importance when working with ambulatory or continuous long-term ECG recordings, which are commonly corrupted by the artifacts caused by muscle activity or moving electrodes. Several indices have been proposed for assessing the quality of ECG signals, including the relative power of baseline variation, signal kurtosis, and the ratio of the number of beats detected by two different QRS detectors, of which one detector is tuned to be more sensitive to noise than the other $[1,2]$. These SQIs are wellsuited for assessment of overall signal quality, where the aim is to exclude bad-quality segments from further analysis.

The analysis of atrial fibrillation (AF) is frequently performed in ambulatory settings. The challenge remains to develop methods robust enough to handle the often encountered poor signal quality, in particular when the ECGs are recorded using hand-held, single-lead devices [3]. While the previously mentioned SQIs are of use when performing RR interval analysis, there is a need for an SQI specifically designed to provide information on whether the f-waves can be reliably measured and analyzed. If accurately extracted from the original ECG, the properties of the f-waves may contain clinically relevant information about the disease [4].

This study introduces a novel approach to f-wave modeling, using a harmonic signal accounting for variations of f-wave amplitude and phase. The fundamental frequency is given by the AF rate, estimated from the observed signal using a maximum likelihood approach. The proposed f-wave SQI uses the root-mean-square (RMS) of the difference between the model signal and the extracted f-wave signal.

\section{Method}

As input, the method always uses the complex atrial activity signal $\mathrm{x}$, obtained by applying spatiotemporal QRST cancellation [5], followed by the Hilbert transform, to ECG signals.

\subsection{Modeling of f-waves}

The $\mathrm{f}$-waves are modeled by the complex signal $s(n ; \theta)$, defined as the sum of two complex exponentials with fundamental angular frequency $\omega_{0}$,

$$
\begin{aligned}
s(n ; \theta) & =\sum_{m=1}^{2} A_{m} e^{j\left(m \omega_{0} n+\phi_{m}\right)}, \\
\theta & =\left[\begin{array}{lllll}
\omega_{0} & A_{1} & A_{2} & \phi_{1} & \phi_{2}
\end{array}\right]^{T},
\end{aligned}
$$

where $A_{m}$ and $\phi_{m}$ denote the amplitude and phase, respectively, of the $m$ :th exponential. All parameters are assumed to be deterministic but unknown. The model $\mathrm{f}$-wave signal, having a length of $N$ samples, is represented by the $N \times 1$ vector $\mathbf{s}(\theta)$, in which $s(n ; \theta)$ is the $n$ :th element. 
The input signal $\mathbf{x}$ is assumed to be the sum of $\mathbf{s}(\theta)$ and white, complex Gaussian noise. Thus, $\mathbf{x}$ is characterized by the following probability density function

$$
p(\mathbf{x}, \theta)=\frac{1}{\pi^{\mathbf{N}} \sigma^{\mathbf{2 N}}} \mathbf{e}^{-\frac{\|\mathbf{x}-\mathbf{s}(\theta)\|^{2}}{\sigma^{2}}},
$$

where $\|\cdot\|^{2}$ denotes the Euclidian norm and $\sigma^{2}$ denotes the noise variance, which initially is assumed to be known. The maximum likelihood estimator of $\theta$ can therefore be formulated as

$$
\hat{\theta}=\underset{\theta}{\arg \min }\|\mathbf{x}-\mathbf{s}(\theta)\|^{\mathbf{2}}
$$

where the estimate of $\omega_{0}$ is constrained to realistic AF frequencies. The optimization is performed using the white noise approach described in [6]. The estimate $\hat{\omega}_{0}$ represents a global frequency estimate as it is based on the entire signal, and can be regarded as an estimate of the AF frequency.

Since f-waves may exhibit considerable variation in AF frequency [7], variation in $\omega_{0}$ is allowed by dividing $\mathbf{x}$ into $K$ overlapping subsegments and determining a local frequency estimate $\hat{\omega}_{0, k}$, which may deviate a maximum of $\Delta \omega_{0}$ from $\hat{\omega}_{0}$, for each subsegment, using the same maximum likelihood approach as before.

The local frequency and phase estimates $\hat{\omega}_{0, k}$ and $\hat{\phi}_{m, k}$ are used to create constant-amplitude basis vectors $\mathbf{b}_{\mathbf{m}}, m=$ 1,2 , describing the phase variation of the signal, by computing the local phase information vectors $\mathbf{y}_{\mathbf{m}, \mathbf{k}}$,

$$
\mathbf{y}_{\mathbf{m}, \mathbf{k}}=\left[\begin{array}{c}
m \hat{\omega}_{0, k} 0+\hat{\phi}_{m, k} \\
m \hat{\omega}_{0, k} 1+\hat{\phi}_{m, k} \\
\vdots \\
m \hat{\omega}_{0, k}(L-1)+\hat{\phi}_{m, k}
\end{array}\right], \quad \mathbf{k}=\mathbf{1}, \ldots, \mathbf{K},
$$

which contains phase information of the $m$ :th harmonic in the $k$ :th subsegment. The harmonically related phase vectors $\mathbf{y}_{\mathbf{m}, \mathbf{k}}$ are aligned and averaged to produce a global $N \times 1$ phase vector $\mathbf{y}_{\mathbf{m}}$, used to construct a basis vector $\mathbf{b}_{\mathbf{m}}$ for each harmonic,

$$
\mathbf{b}_{\mathbf{m}}=\cos \left(\mathbf{y}_{\mathbf{m}}\right)
$$

The model signal is finally obtained by multiplying each element of each basis vector, $b_{m}(n)$, with the corresponding sample-by-sample amplitude $a_{m}(n)$. The amplitudes are estimated by imposing a constraint $\Delta a_{m}$ on the maximum sample-to-sample variation in $a_{m}(n)$,

$$
\left|a_{m}(n)-a_{m}(n-1)\right| \leq \Delta a_{m}, \quad n=1, \ldots, N-1,
$$

and minimizing the error between the model signal and the input signal using a least squares estimator.

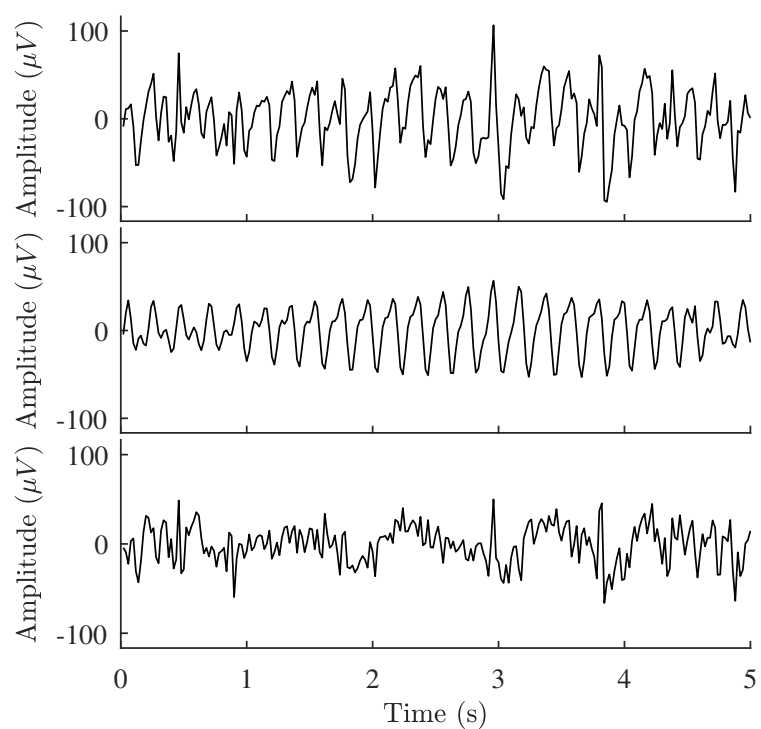

Figure 1. Example of f-wave modeling including a) observed $\mathbf{f}$-waves $\mathbf{x}, \mathrm{b}$ ) modeled $\mathrm{f}$-waves $\hat{\mathbf{s}}$ and $\mathrm{c}$ ) model error $\mathbf{x}-\hat{\mathbf{s}}$.

The minimization problem is solved using the convex optimization package CVX (CVX Research, Inc [8]). Finally, the estimated model signal $\hat{s}(n)$ is obtained by multiplying the estimated amplitudes with the basis functions,

$$
\hat{s}(n)=\sum_{m=1}^{2} \hat{a}_{m}(n) b_{m}(n), \quad n=1, \ldots, N
$$

An f-wave modeling example is presented in Fig. 1.

\subsection{Signal quality index}

The normalized RMS of the model error $\mathbf{x}-\hat{\mathbf{s}}$ defines the SQI,

$$
S=1-\frac{\sigma_{\mathbf{x}-\hat{\mathbf{s}}}}{\sigma_{\mathbf{x}}}
$$

where $\sigma_{\mathbf{x}-\hat{\mathbf{s}}}$ and $\sigma_{\mathbf{x}}$ denote the RMS of $\mathbf{x}-\hat{\mathbf{s}}$ and $\mathbf{x}$, respectively. The value of $\mathcal{S}$ is restricted to the interval $[0,1]$, where 0 indicates poor signal quality and 1 indicates perfect modeling of $\mathbf{x}$.

\subsection{Datasets and Performance Evaluation}

A database with 12-lead ECGs recorded from 189 patients with permanent AF is analyzed, acquired at the University Hospital in Lund [9]. A 5-s segment is extracted from lead $V_{1}$ in each ECG. Spatiotemporal QRST cancellation is performed to obtain the atrial activity signals.

Two types of noise, muscle artifacts and noise due to electrode movements, are extracted from the MIT-BIH 
Table 1. Simulation parameters for the sawtooth f-wave model.

\begin{tabular}{|l|l|}
\hline \multicolumn{2}{|c|}{ Simulation setup } \\
\hline$H$ & 3 \\
$F_{0}$ & $7 \mathrm{~Hz}$ \\
$\Delta F$ & $0.25 \mathrm{~Hz}$ \\
$F_{m}$ & $0.2 \mathrm{~Hz}$ \\
$\Delta a$ & $a / 3$ \\
$F_{a}$ & $0.2 \mathrm{~Hz}$ \\
\hline
\end{tabular}

Noise Stress Test Database [10]. The signal-to-noise ratio (SNR) is defined by

$$
\mathrm{SNR}=\frac{\sigma_{f}}{\sigma_{n}}
$$

where $\sigma_{f}$ and $\sigma_{n}$ denotes the RMS of the f-waves and the RMS of the noise, respectively. To evaluate the relationship between $\mathcal{S}$ and SNR, this study uses an extended version of the sawtooth model for simulating f-waves proposed in [5], which includes a stochastic component so that more complex f-wave patterns can be generated [11]. The simulated f-waves are composed of two components with equal RMS,

$$
f(t)=f_{d}(t)+f_{s}(t),
$$

where $f_{d}(t)$ is the sawtooth signal defined by

$$
f_{d}(t)=\sum_{h=1}^{H} a_{h}(t) \sin \left(2 \pi h F_{0} t+h \frac{\Delta F}{F_{m}} \sin \left(2 \pi F_{m} t\right)\right),
$$

where $F_{0}$ denotes the AF frequency, $\Delta F$ denotes the maximum frequency deviation, and $F_{m}$ denotes the modulation frequency. The amplitude $a_{h}(t)$ is defined by

$$
a_{h}(t)=\frac{2}{h \pi}\left(a+\Delta a \sin \left(2 \pi F_{a} t\right)\right), \quad h=1, \ldots, H,
$$

where $a$ denotes the sawtooth amplitude, $\Delta a$ the maximum amplitude deviation, and $F_{a}$ the amplitude modulation frequency. The stochastic component $f_{s}(t)$ results from bandpass filtering of white noise - the filter having two passbands symmetrically related to $F_{0}$ by $\left[0.65 F_{0}, 0.95 F_{0}\right]$ and $\left[1.05 F_{0}, 1.35 F_{0}\right]$. The parameter $a$ is chosen such that $\sigma_{f}=50 \mu \mathrm{V}$. The remaining simulation parameters are displayed in Table 1. The continuous signal $f(t)$ is sampled at a rate of $50 \mathrm{~Hz}$. Examples of simulated signal with different SNRs are illustrated in Fig. 2.

Prior to signal quality assessment, the original sampling rates are decimated to $50 \mathrm{~Hz}$ and the segment length $N$ is set to 250 samples, i.e., $5 \mathrm{~s}$. The signal subsegments have a length of $0.5 \mathrm{~s}$, thus containing at least one fwave period. Each subsegment is shifted one sample at a time from the preceding subsegment. The grid search

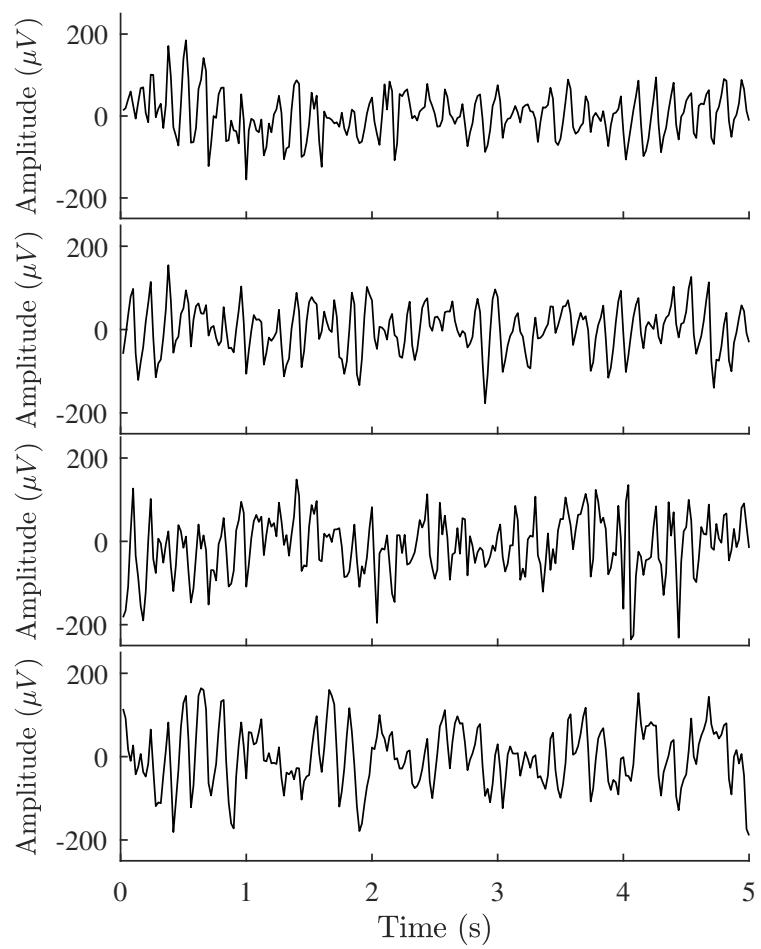

Figure 2. Examples of simulated f-waves with added noise from a) motion artifacts, $\mathrm{SNR}=2$, b) electrode movements, $\mathrm{SNR}=2, \mathrm{c})$ motion artifacts, $\mathrm{SNR}=1$ and d) electrode movements, $\mathrm{SNR}=1$.

for finding the global estimate $\hat{\omega}_{0}$ is performed in the interval $[4,12] \mathrm{Hz}$. Moreover, the maximum deviation is set to $\Delta \omega_{0}=0.25 \mathrm{~Hz}$. The maximum sample-to-sample variation in amplitude is set to $1 \%$ of the RMS of the input signal for the fundamental frequency $\left(\Delta a_{0}=0.01 \sigma_{x}\right)$, whereas it is set to zero for the harmonic $\left(\Delta a_{1}=0\right)$.

\section{Results}

Figure 3 presents the distribution of $\mathcal{S}$ for f-wave signals in the 189 5-s segments from the QRST-cancelled ECGs, along with the distributions of $\mathcal{S}$ for equally many signals containing either muscle artifacts or noise due to electrode movements (but without cardiac activity). Both types of noise are characterized by considerably lower values of $\mathcal{S}$ than are f-waves and $\mathcal{S}$ is capable of discriminating between f-waves and noisy recordings with an accuracy at $98 \%$.

The relationship between $\mathcal{S}$ and SNR is established for muscle artifacts and noise due to electrode movements added to simulated f-waves. In total, $\mathcal{S}$ is computed for 10000 different realisations of f-waves and noise. The results are presented in Fig. 4. For $\mathrm{SNR}=1, \mathcal{S}$ is close to 0.3 , being slightly larger for muscle artifacts than for noise due to electrode movements. As the SNR decreases, 


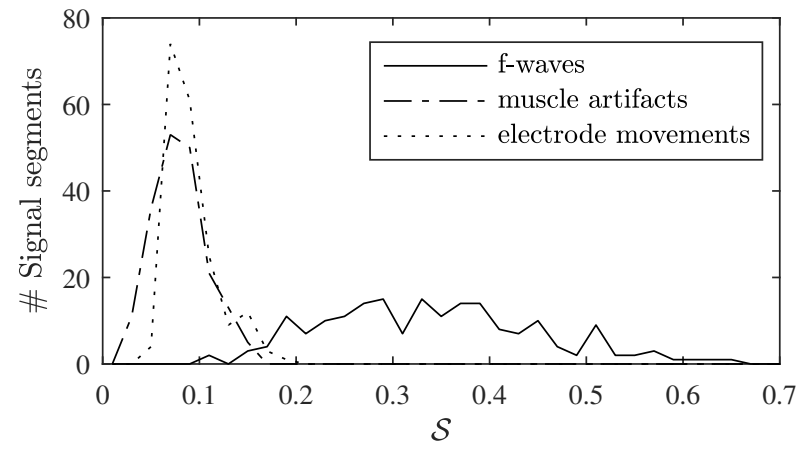

Figure 3. Histograms of $\mathcal{S}$ for signals with either f-waves, muscle artifacts, or noise due to electrode movements. For ease of interpretation, the histogram bins are connected with straight lines.

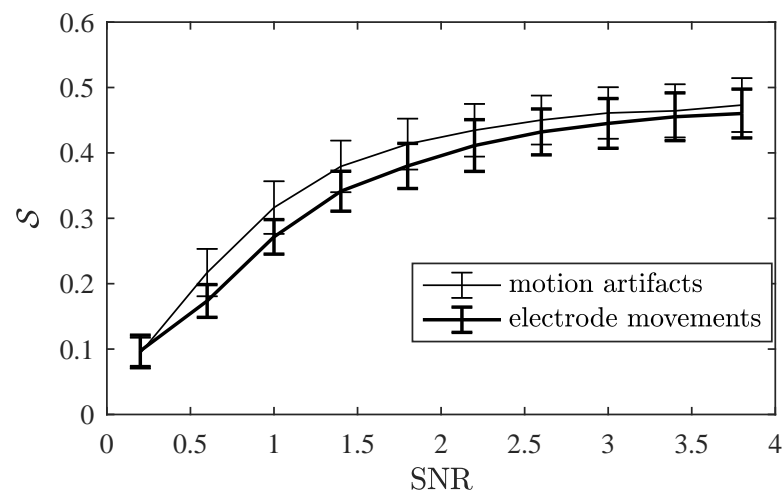

Figure 4. $\mathcal{S}$ as a function of SNR. The bars represent the standard deviation.

$\mathcal{S}$ obtains a value of about 0.1 , i.e., similar to the values for the noisy signals in Fig. 3. It should be noted that the dispersion of $\mathcal{S}$ is largely independent of SNR.

\section{Discussion and Conclusion}

A novel f-wave SQI for use in QRST-cancelled ECG signals is introduced. The computation of $\mathcal{S}$ is performed using a harmonic $\mathrm{f}$-wave model based on the maximum likelihood estimate of fundamental frequency and allowing for variations of amplitude and phase. When applied to ECG signal from AF patients and noise signals from the MIT-BIH Noise Stress Test Database, $\mathcal{S}$ successfully separates the two signal types with high accuracy.

The value of $\mathcal{S}$ is also clearly responsive to increases in noise level in signal containing f-waves, which is demonstrated in Fig. 4. This suggests that the use of $\mathcal{S}$ assists in the exclusion of noisy AF signals, and thus improves the reliability and robustness of the subsequent analysis of the f-waves.

The properties of $\mathcal{S}$ should be further studied, in par- ticular with respect to ECG signals not containing AF, e.g. from patients in sinus rhythm or in other arrhythmias. Also, a more thorough study regarding its relation to $\mathrm{f}$-wave properties, and the accuracy of estimating these properties, is needed.

\section{References}

[1] Clifford GD, Behar J, Li Q, Rezek I. Signal quality indices and data fusion for determining clinical acceptability of electrocardiograms. Physiol Meas 2012;33(9):1419.

[2] Behar J, Oster J, Li Q, Clifford GD. ECG signal quality during arrhythmia and its application to false alarm reduction. IEEE Trans Biomed Eng 2013;60(6):1660-1666.

[3] Mehta DD, Nazir NT, Trohman RG, Volgman AS. Singlelead portable ECG devices: Perceptions and clinical accuracy compared to conventional cardiac monitoring. J Electrocardiol 2015;48(4):710-716.

[4] Petrutiu S, Ng J, Nijm GM, Al-Angari H, Swiryn S, Sahakian AV. Atrial fibrillation and waveform characterization. IEEE Eng Med Biol Mag 2006;25(6):24-30.

[5] Stridh M, Sörnmo L. Spatiotemporal QRST cancellation techniques for analysis of atrial fibrillation. IEEE Trans Biomed Eng 2001;48(1):105-111.

[6] Christensen MG, Jensen SH. Variable order harmonic sinusoidal parameter estimation for speech and audio signals. In Proc. 40th Asilomar Conf. Signals, Systems Comput. IEEE, 2006; 1126-1130.

[7] Sandberg F, Bollmann A, Husser D, Stridh M, Sörnmo L. Circadian variation in dominant atrial fibrillation frequency in persistent atrial fibrillation. Physiol Meas 2010; 31(4):531.

[8] Grant M, Boyd S. CVX: Matlab software for disciplined convex programming, version 2.1, March 2014.

[9] Stridh M, Sörnmo L, Meurling CJ, Olsson SB. Sequential characterization of atrial tachyarrhythmias based on ECG time-frequency analysis. IEEE Trans Biomed Eng 2004; 51(1):100-114.

[10] Goldberger A, Amaral L, Glass L, Hausdorff J, Ivanov P, Mark R, Mietus J, Moody G, Peng CK, Stanley H. Physiobank, Physiotoolkit, and Physionet: Components of a new research resource for complex physiologic signals. Circulation 2000;101(23):215-220.

[11] Petrènas A, Marozas V, Sörnmo L, Lukoševičius A. An echo state neural network for QRST cancellation during atrial fibrillation. IEEE Trans Biomed Eng 2012; 59(10):2950-2957.

Address for correspondence:

Mikael Henriksson

Department of Biomedical Engineering, Lund University,

SE-22100 Lund, Sweden

mikael.henriksson@bme.lth.se 